Tropical Journal of Pharmaceutical Research December 2012; 11 (6): 909-916

(C) Pharmacotherapy Group,

Faculty of Pharmacy, University of Benin

Benin City, 300001 Nigeria

All rights reserved.

Available online at http://www.tjpr.org

Research Article

http://dx.doi.org/10.4314/tjpr.v11i6.6

\title{
Over-expression of NAD kinase in Corynebacterium crenatum and its Impact on L-Arginine Biosynthesis
}

\author{
Muhammad Masfiqur Rahman ${ }^{1}$, Zhao Qin Qin ${ }^{1}$, Wenfang Dou ${ }^{1}$, Rao \\ Zhiming ${ }^{1}$ and Zhenghong $\mathrm{Xu}^{1,2^{*}}$ \\ ${ }^{1}$ Laboratory of Pharmaceutical Engineering, School of Medicine and Pharmaceutics, ${ }^{2}$ The Key Laboratory of \\ Industrial Biotechnology, Ministry of Education, School of Biotechnology, Jiangnan University, Wuxi 214122, P R \\ China
}

\begin{abstract}
Purpose: To improve the biosynthesis of L-arginine by overexpressing homologous NAD kinase (ppnk) in Corynebacterium crenatum SYPA5-5 and to study its impact in presence of high (HOS) and low oxygen supply (LOS).

Methods: A recombinant plasmid (pJC1-tac-ppnK) harboring homologous NAD kinase (ppnk) was constructed in a shuttle vector pJC1 and transferred in L-arginine producing strain Corynebacterium crenatum SYPA5-5. Furthermore, fermentation was performed by shake flask method with consecutive determination of cell growth and glucose concentration. $N A D^{+}$kinase activity was studied by stop method and $N A D P(H)$ concentrations were determined by spectrophotometric enzymatic cycling method. To check the biosynthesis of amino acids, HPLC method was used to determine extracellular amino acid concentrations.

Results: In HOS condition, $\mathrm{NAD}^{+}$kinase activity increased by $116 \%$, while intracellular concentrations of $N A D P^{+}$and $N A D P H$ increased by 7.3 and $36.8 \%$, respectively. Whereas, in LOS condition, $N A D^{+}$ kinase activity increased $49 \%$, with intracellular 14.67 and $15 \%$ increases in NADP ${ }^{+}$and NADPH respectively. More importantly, recombinant strain could produce 26.47 and $11.36 \mathrm{~g} / \mathrm{L}$ L-arginine in HOS and LOS respectively, which is higher than control strain value of 24.29 and $7.58 \mathrm{~g} / \mathrm{L}$ respectively.

Conclusion: These results suggest that altering the concentration of co-enzymes by NAD kinase in Corynebacterium crenatum is an effective way to increase $N A D P^{+}$with concurrent production of NADPH for further enhanced L-arginine biosynthesis in Corynebacterium crenatum in both conditions of high and low oxygen supply.
\end{abstract}

Keywords: NAD kinase, PpnK, L-arginine, Corynebacterium crenatum 


\section{INTRODUCTION}

L-Arginine (Arg) is classified conditionally as an essential amino acid for adults and essential amino acid for birds, carnivores and young mammals. L-arginine administration reverses endothelial dysfunction, enhances wound healing, prevents the early stages of tumorigenesis, and improves cardiovascular, reproductive, pulmonary, renal, digestive, and immune functions [2].

Nicotinamide adenine dinucleotide phosphate (NADPH), an important co-enzyme during anabolic reactions plays a significant role during biosynthesis of amino acids in $C$. glutamicum [13]. L-arginine biosynthetic pathways require NADPH for reductive amination of oxoglutarate to glutamate by glutamate dehydrogenase and for the formation of N-Acetylglutamate-5 -semialdehyde by $\mathrm{N}$-acetyl-gamma-glutamylphosphate reductase [19]. It has been reported that in $C$. glutamicum NADPH is generated mainly through $\mathrm{NADP}^{+}$dependent dehydrogenases such as glucose-6phosphate dehydrogenase (G6PDH) and 6phosphogluconate dehydrogenase (6PGDH/ gnd) in oxidative Pentose phosphate pathway (PPP) and partly by NADP dependant isocitrate dehydrogenase (ICD) and NADP dependent malic enzyme (ME/ malE) [33-35]. $\operatorname{NADP}(\mathrm{H})$ could also be formed from the biosynthetic pathway of $\operatorname{NADP}(\mathrm{H})$ by Phosphorylation of NAD through NAD kinase (EC 2.7.1.23), which is a critical enzyme and expected to be essential for the regulation of $\operatorname{NAD}(\mathrm{H})$ and $\operatorname{NADP}(\mathrm{H})$ balance. It has been reported that G6PD is the rate controlling step for PPP and NADPH from oxidative part of PPP in $C$.glutamicum, seems to be essential for the higher production of Lysine biosynthesis. Moreover, kinetic studies on G6PD showed NADPH is a strong inhibitor of G6PD [14,15]. As a result, direct conversion of NADPH might affect the PPP. Presence of multiple NAD kinase genes have been reported in several microorganisms [10,32]. However, in C. glutamicum, only one NAD kinase gene has been reported (ppnK) and seems to be essential for this organism [13]. Very recently, the purified NAD kinase from C. glutamicum ssp. lactofermentum ATCC 13869 showed weak NADH kinase activity besides the $\mathrm{NAD}^{+}$kinase activity [36]. Most of the NAD kinases were found allosteric in nature and due to this fact, the $\mathrm{NAD}(\mathrm{H})$ and $\operatorname{NADP}(H)$ balance might be directly regulated by NAD kinase $[9,10,32]$.

Bacterial fermentation from natural carbon sources is currently the major approach to industrial scale production of L-arginine $[1,3,4]$. Corynebacterium crenatum SYPA5-5 is an aerobic, Gram-positive, non-sporulating, and L-histidine auxotroph industrial bacterium was isolated from soil and mutated by UV in our previous work [7]. As an aerobic bacterium, C. crenatum requires a large amount of dissolved oxygen (DO) for Larginine production and plays an important role for L-arginine production [7].

Altering the level of co-enzyme(s) involved in L-arginine production pathway could be one of the possible approaches for further enhancing L-arginine production. In this present studies we have successfully constructed the homologous NAD kinase in shuttle vector pJC1 (pJC1-tac-ppnK) , overexpressed in $C$. crenarum to further increase the $\mathrm{NADP}^{+}$that might stimulate PPP with concurrent production of NADPH and precursor metabolites. Moreover, the impact of NAD Kinase over-expression on L-arginine fermentation in the presence of high oxygen supply (HOS) and low oxygen supply (LOS) was investigated.

\section{EXPERIMENTAL}

Reagents, Strains, plasmids and oligonucleotide primers

Plasmids were constructed in E. coli JM109 from PCR-generated fragments (ExTaq DNA Polymerase, TaKaRa) using $C$. crenatum SYPA5-5 DNA as a template prepared according to Eikmanns et al. [25]. 
In order to construct pMD19-T-ppnK and pJC1-tac-ppnK, the ppnK gene was amplified by PCR using the upstream primer ppnK $F$ Xba I: 5'-GCTCTAGAATGACTGCACCCAC GAACG-3'; the introduction of a Xba I restriction site was underlined and start codon is highlighted in bold. The downstream primer ppnK $\mathrm{R}$ Sal I: 5'-GCGTCGAC TTACCCCGCTGACCTGG-3'; the introducetion of a Sal I restriction site was underlined; the stop codon is highlighted in bold. The PCR fragment was cloned into the pMD19-T vector (TaKaRa), the obtained plasmid pMD19-T-ppnK was confirmed by digestion with Xba I and Sall, and the Xba I /Sal I fragment of ppnK from pMD19-T-ppnK was then inserted into the corresponding sites of pJC1-tac [8], resulting in plasmid pJC1-tacppnK. C. crenatum SYPA5-5 with an empty vector was used as control. NADP ${ }^{+}$, glucose6-phosphate dehydrogenase, glutamate dehydrogenase, alcohol dehydrogenase, thiazolyl blue were purchased from Sigma (St. Louis, MO, USA). phenazine ethosulphate was from Santa Cruz Biotech( California, USA) .NAD ${ }^{+}, \mathrm{NADPH}, \mathrm{ATP}$, glucose-6- phosphate were from Sangon(Shanghai, China).

\section{Transformation and Screening}

The ligation mixture was used to transform into Escherichia coli by the calcium chloride method [26].Transformation in C. crenatum was performed by using electroporation method and freshly transformed culture was incubated at $46^{\circ} \mathrm{C}$ water bath for 6 min with an additional shaking at $200 \mathrm{rpm}, 30^{\circ} \mathrm{C}$. Finally, transformed culture was incubated on solid LB containing $9.1 \%$ sorbitol at $30^{\circ} \mathrm{C}$ until the colonies appeared $[7,8]$. When necessary, ampicillin (100 $\left.\mu \mathrm{g} \mathrm{mL} \mathrm{m}^{-1}\right)$ or kanamycin $\left(50 \mu \mathrm{g} \mathrm{mL}^{-1}\right)$ was added at final concentration for transformant selections.

\section{Growth medium and conditions for L- arginine production}

Bacteria were cultivated in rich Luria-Bertani (LB) medium, standard for $E$. coli.
Corynebacterium sp were cultivated in LB medium with $0.5 \%$ glucose and for $C$. crenatum competent cell medium, LB including $3 \%$ glycine and $0.1 \%$ Tween was used $[7,26]$. Slant medium, seed medium, and shake flask fermentation medium of $C$. crenatum prepared according to $\mathrm{Xu}[7,27]$.

For L-arginine batch fermentation, C. crenatum strains were activated in slant medium. After $24 \mathrm{~h}$, the seed was inoculated from agar slants and cultured at $30^{\circ} \mathrm{C}$ for about $15 \mathrm{~h}$ in shake flasks. Furthermore, the shake flask seed cultures were transferred into $250 \mathrm{ml}$ flask to final $\mathrm{OD}_{562}=1$; the shake flask feed batch fermentation was performed at $30^{\circ} \mathrm{C}$ for 96 h. For shake flask fermentation, $15 \%$ glucose was added in two steps. Initially $10 \%$ and an additional 5\% glucose were added after $36 \mathrm{~h}$ to the feed batch shake flask fermentation culture. $20 \mathrm{ml}$ fermentation culture in $250 \mathrm{ml}$ flask and $60 \mathrm{ml}$ fermentation culture in $250 \mathrm{ml}$ flask were classified as high oxygen supply (HOS) and low oxygen supply (LOS) respectively [28]. Strain harboring the recombinant plasmid pJC1-tac-ppnK was cultivated with kanamycin $\left(30 \mu \mathrm{g} \mathrm{mL} \mathrm{m}^{-1}\right)$ before being transferred into the shake flask fermentation medium [27].

Assay of cell concentration, glucose, Larginine and other amino acids

Initial cell concentration was monitored at 562 $\mathrm{nm}$, and the dry cell weight (DCW) was determined by a pre-calibrated relationship (1 $\left.\mathrm{OD}=0.375 \mathrm{gL}^{-1} \mathrm{DCW}\right)$ [7]. Glucose concentration in the media was measured by using anthrone method (35). Concentrations of L-arginine and other amino acids were measured using a reversed-phase highpressure liquid chromatography [7]. For the quantification of product formation and glucose concentration, a 200 300 $\mu$ L sample was removed from the culture and was centrifuged at $12,000 \mathrm{rpm}$ for $10 \mathrm{~min}$, further the supernatant was used to determine the amino acid concentration in the culture fluid. All of the measurements, particularly the 
most important state variables, such as the concentrations of cells, L-arginine, and glucose, were measured in triplicate.

\section{Assay of $\mathrm{NAD}^{+}$kinase activity}

Crude cell extracts were prepared from cells grown in fermentation medium as described above. Cells were harvested during the exponential phase by centrifugation $(12,000$ rpm for $5 \mathrm{~min}, 4^{\circ} \mathrm{C}$ ), washed with disruption buffer $(100 \mathrm{mM}$ Tris/ $\mathrm{HCl}, \mathrm{pH} 8.0,10 \mathrm{mM}$ $\mathrm{MgCl} 2$ and $0.75 \mathrm{mM}$ DTT) and disrupted by sonication at $4^{\circ} \mathrm{C}$. Cell debris was removed by centrifugation $(12,000 \mathrm{rpm}$ for $15 \mathrm{~min}$ at $4^{\circ} \mathrm{C}$ ). The obtained clear supernatant was used as a crude enzyme for determination of $\mathrm{NAD}^{+}$kinase activity. $\mathrm{NAD}^{+}$kinase activities were assayed at $30^{\circ} \mathrm{C}$ using a stop method according to $F$. shi et al. [16]. In brief, the formation of NADPH was measured at 340 $\mathrm{nm}$ in a reaction mixture $(1.0 \mathrm{~mL})$ consisting of $5.0 \mathrm{mM}$ NAD, $5.0 \mathrm{mM}$ ATP, $5.0 \mathrm{mM}$ $\mathrm{MgCl}$, $100 \mathrm{mM}$ Tris / $\mathrm{HCl}(\mathrm{pH} \mathrm{8.0)}$ and an appropriate amount of enzyme. Crude enzyme solution of less than $100 \mu \mathrm{L}$ was added to the reaction mixture to initiate the reaction. The reaction was terminated by immersing the tube in boiling water for $5 \mathrm{~min}$, followed by addition of $0.1 \mathrm{~mL}$ of $50 \mathrm{~mm}$ glucose-6-phosphate to the mixture, and the amount of NADP formed was determined enzymatically with $0.5 \cup$ glucose-6phosphate dehydrogenase. Negative controls were carried out without crude enzyme or without ATP, respectively. Enzyme activity (one unit) (U) was defined as $1.0 \mu \mathrm{mol}$ $\mathrm{NADP}^{+}$produced for $1 \mathrm{~min}$ at $30^{\circ} \mathrm{C}$ in the initial mixture $(1.0 \mathrm{ml})$, and the specific activity was expressed as $\mathrm{U} / \mathrm{mg}$ of protein. Bradford method was used to determine protein concentrations with BSA as a standard [24].

\section{Determination of the intracellular NADP(H) concentrations}

The intracellular NADP $(\mathrm{H})$ concentrations were determined according to the method of Shi $F$ et al [36], Crude cell extracts were prepared from cells grown in the fermentation medium of Corynebacterium crenatum and frozen in liquid nitrogen. Cells were washed with cold $100 \mathrm{mM}$ PBS $(\mathrm{pH} 7.5)$ and pellet $\sim 10^{5}$ cells for each sample were maintained. $\mathrm{NADP}^{+}$and NADPH were extracted using $\mathrm{HCl}$ and $\mathrm{NaOH}$, respectively [37]. The amounts of $\mathrm{NADP}^{+}$and NADPH in each extract were quantified using a spectrophotometric enzymatic cycling method with an NADP ${ }^{+}$-specific glucose-6-phosphate dehydrogenase (G6PDH) and an NAD specific yeast alcohol dehydrogenase (ADH). The reactions were carried out at $25^{\circ} \mathrm{C}$ and were initiated by the addition of the cell extract. The absorbance at $570 \mathrm{~nm}$ was followed for $5 \mathrm{~min}$ to determine the NADP ${ }^{+}$ and NADPH concentrations. The concentrations were calculated using external $\mathrm{NADP}^{+}$and NADPH standards and with the intracellular volume of $1.6 \mu \mathrm{L} / \mathrm{mg}$ dry weight.

\section{RESULTS}

\section{Effects of homologues NAD kinase overexpression on Cell growth and glucose consumption}

NAD kinase had slight negative effect on cell growth during the overall $108 \mathrm{~h}$ fermentation period (Figs $1 \mathrm{~A}$ and 2) In case of LOS condition the control and recombinant strain produced 21 and $17 \mathrm{~g} / \mathrm{L}$, respectively (Fig 2A) which is significantly lower when compared with HOS where the control and recombinant strain produced 32 and $27 \mathrm{~g} / \mathrm{L}$, respectively (Fig 1A). After $36 \mathrm{~h}$ of fermentation $40 \%$ glucose was consumed by recombinant stain which is lower than control strain where $50 \%$ glucose was consumed.

\section{Enzymatic activity of $\mathrm{NAD}^{+}$kinase and intracellular NADP $(H)$ concentration}

Enzymatic assay illustrates that $\mathrm{NAD}^{+}$kinase activities in the recombinant strain harboring homologous NAD kinase gene increased in the both HOS and LOS conditions by 116 and $49 \%$, respectively, when compared to the control strain. (Fig 3). At HOS, 
concentrations increased 7.3 and $36.8 \%$ in $\mathrm{NADP}^{+}$and NADPH respectively, whereas, at LOS, concentration were increased 14.7 and $15.0 \%$ in $\mathrm{NADP}^{+}$and NADPH, respectively (Table 1).
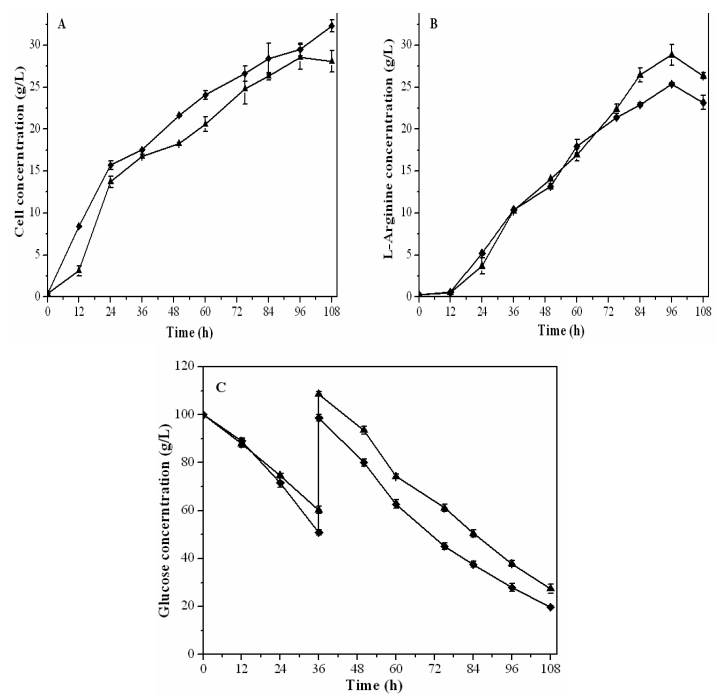

Fig 1: Cell growth, glucose consumption and Larginine concentration from fermentation medium of $C$. crenatum under HOS condition. (A) Cell concerntration; (B) L-Arginine concerntration; (C) glucose concerntration; ( $\mathbf{\Delta})$ Recombinant strain; (४) control strain

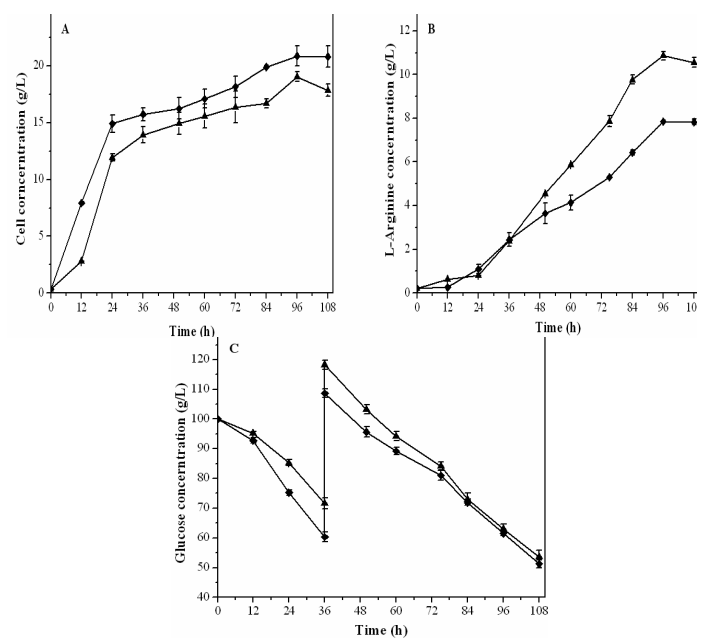

Fig 2: Cell growth, glucose consumption and Larginine concentration from fermentation medium of $C$. crenatum under LOS condition. (A) Cell concerntration; (B) L-arginine concerntration; (C) glucose concerntration. ( $\mathbf{\Lambda})$ Recombinant strain; (४) Control strain

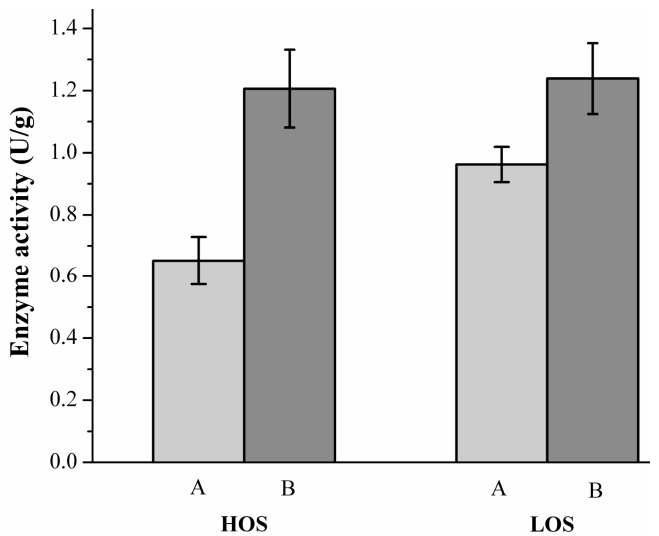

Fig 3: Comparison of $\mathrm{NAD}^{+}$kinase activities under HOS and LOS conditions. (A) Control strain. (B) Recombinant strain.

Table 1: Intracellular concentration $(\mu \mathrm{M})$ of $\mathrm{NADP}^{+}$and NADPH in C. crenatum

\begin{tabular}{lcclcc}
\hline & \multicolumn{2}{c}{ NADP $^{+}$} & & \multicolumn{2}{c}{ NADPH } \\
\cline { 2 - 3 } \cline { 5 - 6 } & HOS & LOS & & HOS & LOS \\
\hline SYPA 5-5 & $739 \pm 17$ & $545 \pm 13$ & & $19 \pm 2$ & $20 \pm 1$ \\
SYPA5-5 / & $793 \pm 18$ & $627 \pm 28$ & & $26 \pm 2$ & $21 \pm 1$ \\
NADK & & & & \\
\hline
\end{tabular}

\section{Improved L-arginine biosynthesis through NAD kinase overexpression}

The C. crenatum recombinants and control strains were fermented to produce L-arginine and extracellular amino acid concentrations were detected from the fermentation medium, according to the procedure described in material and methods section. For HOS condition, till $72 \mathrm{~h}$ of fermentation, L-arginine biosynthesis of recombinant strain was almost same with control strain. However, during 72 to $108 \mathrm{~h}$ the L-arginine biosynthesis was increased for recombinant strain (Fig 1 B). In contrast, in LOS condition, after $36 \mathrm{~h}$ of fermentation, the L-arginine biosynthesis of recombinant strain was increased significantly compared to control strain (Fig 2 $B)$. The results demonstrated that presence of recombinant plasmid pJC1-tac-ppnK in C. crenatum SYPA5-5 could enhance L-arginine production, despite the fact that the NAD kinase gene had a negative effect on cell 
growth in presence of HOS. Recombinant strain synthesis $26.47 \mathrm{~g} / \mathrm{L} \quad \mathrm{L}$-arginine compared to control strain $24.29 \mathrm{~g} / \mathrm{L}$. Besides L-arginine, other byproducts amino acids Llysine and L-isoleucine yield were also improved. It has been reported that NADPH plays a significant role for L-lysine and Lisoleucine biosynthesis $[13,36]$. However, for LOS, recombinant stain could yield $11.36 \mathrm{~g} / \mathrm{L}$ compared to control strain $7.58 \mathrm{~g} / \mathrm{L}$, which is severely lowered than HOS condition (Table 2).

NAD kinase overexpression enhanced the intracellular concentration of $\mathrm{NADP}^{+}$and altered the co-enzymes in the cell and this might cause the negative cell growth for recombinant strain. Glucose was used as a sole carbon source for the fermentation. As the glucose was introduced into two steps, initially at starting of the fermentation and after $36 \mathrm{~h}$ of the fermentation, an increased peak at $36 \mathrm{~h}$ is observed in the graph of glucose consumption. Both cases of HOS and LOS showed the lower consumption rate of glucose for recombinant stain compared with control. However, during the 72 to $108 \mathrm{~h}$ of fermentation, the glucose consumption rate for recombinant and control was almost the same for LOS conditions. In all, the glucose consumption rate in the recombinant stains was retarded during HOS and LOS fermentation that could be due to the decreased cell mass formation.

$\mathrm{NADP}^{+}$and NADPH concentrations increased in recombinant strain harboring NAD kinase gene. It has been reported that NADK regulation can easily allow an organism to switch between a reductive state and an oxidative state [5]. NADPH quantification may vary due to the variations of state of cells [34]. NADPH is required in many pathways including fighting against stressed conditions [20]. It has been reported that the respiratory chain in $C$. glutamicum could also be a potential route for NADPH consumption [38].

L-arginine and other by products amino acids (L-lysine and L-isoleucine) production was increased in presence of HOS. In contrary, Larginine production was perturbed severely in presence of LOS.The interactions between the metabolic reactions and by product formation depend on dissolved oxygen (DO) levels [11]. The low yield of L-arginine in oxygen limiting condition could be due to the suppression of the genes involved in Larginine production. Interestingly, homologous NAD kinase expression could increase amino acid yield in oxygen limiting condition. It seems likely that enhanced $\mathrm{NADP}^{+}$and NADPH concentration could play a significant role in L-arginine production.

\section{CONCLUSION}

Direct pathway engineering and mutageneses are common practices for amino acid biosynthesis. However, recently several researchers have reported improved biosynthesis of amino acids and metabolites by co-factor engineering $[13,36]$. Until now, metabolic networks that produce and

Table 2: Production of L-arginine and other amino acids by $C$. crenatum under different conditions of oxygen supply. Samples were taken from fermentation medium at $96 \mathrm{~h}$.

\begin{tabular}{ccccc}
\hline & \multicolumn{2}{c}{ HOS } & \multicolumn{2}{c}{ LOS } \\
\cline { 2 - 5 } Concentration & \multicolumn{3}{c}{ SYPA 5- } & SYPA 5- \\
\cline { 2 - 5 } (g/L) & SYPA 5-5 & 5/NADK & SYPA 5-5 & 5/NADK \\
\hline Arg & $24.29 \pm 0.40$ & $26.47 \pm 0.37$ & $7.58 \pm 0.790$ & $11.36 \pm 0.14$ \\
Ile & $1.38 \pm 0.00$ & $1.55 \pm 0.04$ & $0.89 \pm 0.13$ & $1.50 \pm 0.020$ \\
Lys & $1.65 \pm 0.01$ & $1.82 \pm 0.05$ & $1.44 \pm 0.12$ & $2.60 \pm 0.06$ \\
\hline
\end{tabular}


maintain the balance of $\mathrm{NAD}^{+}, \mathrm{NADH}$, $\mathrm{NADP}^{+}$, NADPH is still not clear in Corynebacterium sp. Several factors including oxygen supply, NADPH availability influence the L-arginine production. Despite the fact that altering the balance of coenzymes may affect the central metabolic pathways [36], in this paper, we have demonstrated that overexpression of homologous ppnK gene in Corynebacterium crenatum SYPA5-5 has increased the NAD ${ }^{+}$ kinase activity, intracellular $\mathrm{NADP}^{+}$and NADPH concentrations along with increased L-arginine biosynthesis in both conditions of high oxygen supply and low oxygen supply.

\section{ACKNOWLEDGMENT}

This work was supported by Programs for New Century Excellent Talents in University (no. NCET-07-0380, NCET-10-0459), National Basic Research Program (973 Program) (no. 2007CB707804), and the National High-Tech Programs of China (no. 2006AA020104, 2006AA020301, 2007AA02Z 207).

\section{Contribution note}

ZQQ contributed to the work as much as the first-named author and has the status of cofirst author

\section{REFERENCES}

1. Leuchtenberger $W$, Huthmacher K, Drauz $K$. Biotechnological production of amino acids and derivatives: current status and prospects. J. Appl. Microbiol. Biotech. 2005; 69(1): 1-8.

2. Flynn NE, Meininger CJ, Haynes TE, Wu G. The metabolic basis of arginine nutrition and pharmacotherapy. J. Biomed \& Pharmacother 2002; 56: 427-438.

3. Lu C D. Pathways and regulation of bacterial arginine metabolism and perspectives for obtaining arginine overproducing strains. J. Appl. Microbiol. Biotech 2006; 70: 261-272.

4. Utagawa T. Production of arginine by fermentation. J. Nutr 2004; 134: 2854S-2857S.

5. Singh R, Mailloux R J, Puiseux-Dao S, Appanna V $D$. Oxidative stress evokes a metabolic adaptation that favors increased NADPH synthesis and decreased NADH production in
Pseudomonas fluorescens. J. Bact 2007; 189: 6665-6675.

6. Ikeda M, Amino acid production processes. Adv Biochem Eng Biotechnol 2003; 79: 1-35.

7. Xu H, Dou W, Zhang X, Rao Z, Shi Z, Xu Z. A twostage oxygen supply strategy for enhanced Larginine production by Corynebacterium crenatum based on metabolic fluxes analysis. Biochemical Eng. J 2009; 43: 41-51.

8. Xu M, Rao Z, Xu H, Lan C. Dou W, Zhang X, Jin J, $X u Z$ Z. Enhanced production of L-arginine by expression of Vitreoscilla hemoglobin using a novel expression system in Corynebacterium crenatum. Appl. Biochem. Biotech 2011; 163 : 707-719.

9. Grose JH, Joss L, Velick SF, Roth JR. Evidence that feedback inhibition of NAD kinase controls responses to oxidative stress. J Proc Natl Acad. Sci 2006; 103: 7601-7606.

10. Shi $F$, Li Y, Wang $X$. Molecular properties, functions, and potential applications of NAD kinases. ABBS 2009; 41: 352-361.

11. Maghnouj A, Abu - Bakr AAW, Baumberg S, Stalon $V$, Wauven C. Regulation of anaerobic arginine catabolism in Bacillus licheniformis by a protein of the Crp/Fnr family. FEMS Microbiol. Lett 2000; 191: 227-234.

12. Imlay JA, Linn S. Bimodal pattern of killing of DNArepair-defective or anoxically grown Escherichia coli by hydrogen peroxide. J. Bact. 1986; 166: 519-527.

13. Lindner $S N$, Niederholtmeyer $H$, Schmitz $K$, Schoberth SM, Wendisch VF. Polyphosphate/ATP-dependent NAD kinase of Corynebacterium glutamicum: biochemical properties and impact of ppnK overexpression on lysine production. J. Appl. Microbiol. Biotech 2010; 87: 583-593.

14. Moritz B, Striegel K, de Graaf AA, Sahm H. Kinetic properties of the glucose - 6 - phosphate and 6 phosphogluconate dehydrogenases from Corynebacterium glutamicum and their application for predicting pentose phosphate pathway flux in vivo. Eur. J. Biochem 2000; 267: 3442-3452.

15. Moritz $B$, Striegel $K$, de Graaf $A$, Sahm $H$. Changes of Pentose Phosphate Pathway Flux in Vivo in Corynebacterium glutamicum during Leucine-Limited Batch Cultivation as Determined from Intracellular Metabolite Concentration Measurements. J. Metab Eng 2002; 4: 295-305.

16. Shi F, Kawai S, Mori S, Kono E, Murata K. Identification of ATP - NADH kinase isozymes and their contribution to supply of NADP $(H)$ in Saccharomyces cerevisiae. FEBS J 2005; 272: 3337-3349.

17. Eikmanns B J, Rittmann D, Sahm H. Cloning, sequence analysis, expression, and inactivation of the Corynebacterium glutamicum icd gene encoding isocitrate dehydrogenase and biochemical 
characterization of the enzyme. J. Bact 1995; 177: 774-782.

18. Gourdon P, Baucher MF, Lindley ND, Guyonvarch A. Cloning of the Malic Enzyme Gene from Corynebacterium glutamicum and Role of the Enzyme in Lactate Metabolism. J. Appl. Env. Microbiol 2000; 66: 2981-2987.

19. Börmann ER, Eikmanns BJ, Sahm H. Molecular analysis of the Corynebacterium glutamicum gdh gene encoding glutamate dehydrogenase. Mol. Microbiol 1992; 6: 317-326.

20. Brioukhanov $A L$, Netrusov $A I$. Catalase and superoxide dismutase: distribution, properties, and physiological role in cells of strict anaerobes. Biochem. (Moscow) 2004; 69: 949962.

21. Giró M, Carrillo N, Krapp AR. Glucose-6-phosphate dehydrogenase and ferredoxin-NADP $(H)$ reductase contribute to damage repair during the soxRS response of Escherichia coli. J. Microbiol 2006; 152: 1119-1128.

22. Lee $S M$, Koh $H J$, Park $D C$, Song $B J$, Huh $T L$, Park J W. Cytosolic NADP+-dependent isocitrate dehydrogenase status modulates oxidative damage to cells. Free Radical Bio. Med 2002; 32: 1185-1196.

23. Stansen C, Uy D, Delaunay S, Eggeling L, Goergen $J$, Wendisch VF. Characterization of a Corynebacterium glutamicum lactate utilization operon induced during temperature-triggered glutamate production. Appl. Env. Microbiol 2005; 71: 5920-5928.

24. Bradford MM. A rapid and sensitive method for the quantitation of microgram quantities of protein utilizing the principle of protein-dye binding. Anal Biochem 1976; 72: 248-254.

25. Eikmanns BJ, Thum-Schmitz N, Eggeling $L$, Lüdtke KU, Sahm H. Nucleotide sequence, expression and transcriptional analysis of the Corynebacterium glutamicum gltA gene encoding citrate synthase. J. Microbiol 1994; 140: 1817-1828.

26. Kirchner O, Tauch A. Tools for genetic engineering in the amino acid-producing bacterium Corynebacterium glutamicum. J. Biotech 2003; 104: 287-299.

27. Xu M, Dou W, Cai D, Zhang X, Rao Z, Xu Z. Improvement of I-Arginine Production by Overexpression of a Bifunctional Ornithine Acetyltransferase in Corynebacterium crenatum. Appl. Biochem. Biotech 2011: 1-11.

28. Tao S, Dou W, Zhang $X, X u H$, Rao Z, Xu Z. A preliminary study on differential proteomics of
Corynebacterium crenatum in two oxygen supply models 2011; CIESC J. (Chinese)

29. Sauer U, Canonaco F, Heri S, Perrenoud A, Fischer $E$. The soluble and membrane-bound transhydrogenases UdhA and PntAB have divergent functions in NADPH metabolism of Escherichia coli. J. Biological. Chem 2004; 279: 6613-6619.

30. Ying $W$. $N A D+/ N A D H$ and $N A D P+/ N A D P H$ in cellular functions and cell death: regulation and biological consequences. Antioxidants \& redox signaling 2008; 10: 179-206.

31. Young D, Tallman $M$, Landy $K$, Young $T$, Lukas $D$, Lewis B, McGuinness E. Ocular lens NAD kinase: partial purification and metabolic implications. J. Biochem Biophys Res Comm 1998; 247: 154-158.

32. McGuinness ET, Butler JR. NAD+ kinase--a review. Int. J. Biochem 1985; 17: 1-11.

33. Marx $A$, de Graaf $A A$, Wiechert $W$, Eggeling $L$, Sahm H. Determination of the fluxes in the central metabolism of Corynebacterium glutamicum by nuclear magnetic resonance spectroscopy combined with metabolite balancing. J. Biotech. Bioeng 1996; 49: 111 129.

34. Marx A, Eikmanns BJ, Sahm $H$, de Graaf $A A$, Eggeling L. Response of the Central Metabolism in Corynebacterium glutamicum to the use of an NADH-Dependent Glutamate Dehydrogenase. J. Metab Eng 1999; 1: 35-48.

35. Marx A, Striegel K, de Graaf AA, Sahm H, Eggeling L. Response of the central metabolism of Corynebacterium glutamicum to different flux burdens. Biotech. Bioeng 1997; 56: 168-180.

36. Shi $F$, Huan $X$, Wang $X$, Ning J. Overexpression of $N A D$ kinases improves the L-isoleucine biosynthesis in Corynebacterium glutamicum ssp. lactofermentum. Enz Microbial Technol 2012; 51:73-80.

37. Lee $M$, Kim MK, Park YH, Lee SY. Regulatory effects of cellular nicotinamide nucleotides and enzyme activities on poly (3 - hydroxybutyrate) synthesis in recombinant Escherichia coli. Biotechnol. Bioeng 1976; 52: 707-712.

38. Matsushita K,Otofuji A, Iwahashi M, Toyama $H$, Adachi O. NADH dehydrogenase of Corynebacterium glutamicum. Purification of an NADH dehydrogenase II homolog able to oxidize NADPH. FEMS Microbiol. Lett. 2001; 204: $271-276$ 